\title{
Patients as a direct source of information on adverse drug reactions
}

\author{
Andrew S Mitchell, David A Henry, Rob Sanson-Fisher, Dianne L O'Connell
}

\begin{abstract}
To determine whether patients should participate directly in detecting adverse reactions to drugs their ability to provide written reports of symptoms experienced during treatment with amoxycillin or trimethoprim-sulphamethoxazole was investigated. When compared with telephone interviews forms on which patients reported events were reliable (the observed agreement with the same statements posed during telephone calls was $85 \%, x=0.56$ ) and valid (sensitivity $=54 \%$, specificity $=94 \%$ ). Patients were also supplied with forms that invited them to report adverse reactions, and their perceptions were compared with those of a panel of experts, who were informed of all clinical events that had been reported during the detailed telephone interviews. Patients were more conservative than the experts in attributing clinical events to drug treatment. The extent of agreement varied and was notably poor for skin and bowel complaints $(x=0.13$ in each case). The performance of event report forms and reaction report forms as instruments of detection was compared in a hypothetical situation in which the experts' views represented the "truth" about adverse reactions to a new drug. Event reporting had a higher sensitivity than reaction reporting $(42 \% v 24 \%)$ but a lower specificity ( $58 \% v \mathbf{9 8 \%})$.

National centres monitoring adverse drug reactions should probably resist pressure to accept reports of reactions directly from the public, but a system based on large scale reporting of events might be valuable in aiding the early detection of symptomatic reactions to new drugs.
\end{abstract}

\section{Introduction}

Although there have been many attempts to improve the postmarketing surveillance of drugs, ${ }^{1.5}$ patients have participated directly in only a few of them..$^{6-9}$ An extensive centralised monitoring system based on written reports from patients would be cheap and might lead to quicker identification of symptomatic reactions to some new drugs. Such a system would have to be evaluated thoroughly before implementation on any scale. We conducted a pilot study to investigate the reliability and validity of patients reporting. We compared the responses of patients who were asked to report all events experienced during antibiotic treatment with those of patients who were asked to report only what they thought were reactions to the drugs. We also compared patients' perceptions of adverse reactions with those of a panel of experts.

\section{Patients and methods}

The study methods were approved by the regional research ethics committee. We used two types of questionnaire-namely, a reaction report form and the reaction report form combined with an event report form. Community pharmacists were provided with equal numbers of each type arranged in random sequence, and they were asked to distribute them in the predetermined order and after obtaining informed consent to consecutive patients presenting prescriptions for amoxycillin or trimethoprimsulphamethoxazole. The pharmacists were instructed to present the forms to patients as "a survey of the health of people who have to take medicines." They were given no specific advice on counselling patients about adverse drug reactions. Pharmacists made a note of the name and telephone number of all participants and the date of dispensing. Parents completed forms on behalf of children under 12 years old.

The reaction report form, which was given to all patients, requested information on sex and age, why the antibiotic had been prescribed, and whether the drug had been used before. It was a simple version of the type of report card distributed by many national monitoring centres for use by doctors and other health professionals. It invited patients to report anything that they thought was a reaction to their antibiotic during one week of treatment.

The combined forms were arranged with the event report form on the front, which patients were asked to complete first. In the event report form patients were asked to agree or disagree with 33 statements about any disorders of or changes in body systems and senses during the week of treatment. Other than directing patients to parts of the body or systems the statements were open ended-for example, "in the past week there has been something wrong with my skin" or "in the past week there has been a change in my mood." Statements contained in the event report form overlapped slightly to maximise the chance of detecting events in different systems. For the purpose of analysis these statements were combined into 19 symptom groups - for instance, ears and hearing were combined as were eyes and eyesight. We also included statements about the heart and liver. We recognised that patients might not accurately attribute symptoms to abnormalities in these organs, but we wanted to present them with an opportunity to report diagnoses made by others. The patients were asked to describe in their own words the abnormalities or changes that they had identified by agreeing with any of the statements.

Patients returned the forms in prepaid envelopes at the end of one week of treatment. Those who did not reply within three days received a maximum of two reminder telephone calls. A structured telephone interview was conducted with patients who returned completed forms. The statements presented in the event report form were put to all patients and were followed by supplementary questions designed to detect any other clinical events affecting the same body systems. Thus for each respondent, irrespective of the original type of report form, we had a complete list of all events that had occurred during the week of drug treatment. This list was presented to a panel of two experts, a clinical pharmacologist and the medical officer/secretary of the Australian Adverse Drug Reactions Advisory Committee. They were informed of the patient's sex and age, the antibiotic prescribed, and the indications reported but not of the patient's perception of any adverse reactions.

The reliability of the event reporting was assessed by measuring the agreement between the written responses and those obtained to the same statements during the telephone interview. We used the kappa statistic $(x)$ to compare the observed agreement with that which would have been expected by chance. ${ }^{10}$ The validity of event reporting was assessed by calculating its sensitivity and specificity when compared with the full telephone interview, which was taken as the standard." To test the agreement between the
Correspondence to: Mr Mitchell. 
patients' and experts' perceptions of adverse reactions the experts evaluated all events elicited in the telephone interviews, judging each to be probably related to treatment or not. We then examined each patient's reaction report to determine which events the patient had identified as adverse reactions. We analysed the level of agreement between patients and experts for each of the 19 symptom groups by means of the $x$ statistic. ${ }^{10}$

Finally we compared the performances of the two reporting forms in a hypothetical situation. The experts' views of which events were probably related to the treatment were taken as representing the "truth" about adverse reactions to a new drug. We compared the rates of detecting these "true" reactions with the event report forms (did the patient identify an event in the relevant body system?) and the reaction report forms (did the patient identify a reaction in that body system?). The performances of the two forms were expressed as sensitivity and specificity. ${ }^{11}$

\section{Results}

During February and March 1987, 20 community pharmacists distributed 267 questionnaires to consecutive patients presenting prescriptions for amoxycillin (139) or trimethoprim-sulphamethoxazole (128). Altogether 109 out of 136 reaction report forms and 97 out of 131 combined event report and reaction report forms were returned, giving an overall response rate of $77 \%$. A total of 176 respondents $(85 \%)$ were interviewed by telephone. Of the 30 patients who could not be interviewed, 11 did not have a telephone, eight had telephones but could not be contacted, and 11 declined to be interviewed. Table I gives the details

TABLE I-Comparison of patients who received the two reporting forms. Figures are numbers (percentages) of patients

\begin{tabular}{lcc}
\hline & $\begin{array}{r}\text { Event report and reaction report form } \\
(\mathrm{n}=83)\end{array}$ & $\begin{array}{c}\text { Reaction report form only } \\
(\mathrm{n}=93)\end{array}$ \\
\hline Male patients & $25(30)$ & $26(28)$ \\
Prescribed amoxycillin & $42(51)$ & $46(49)$ \\
Previous use of same drug & $47(57)$ & $52(56)$ \\
Indication: & $36(43)$ & $42(45)$ \\
$\quad$ Upper respiratory tract infection & $12(14)$ & $8(9)$ \\
Lower respiratory tract infection & $14(17)$ & $23(25)$ \\
Urinary tract infection & $3(4)$ & $6(7)$ \\
Prophylaxis & $6(7)$ & $7(8)$ \\
Skin infection & $2(2)$ & $3(3)$ \\
Bowel infection & $4(5)$ & $1(1)$ \\
Dental infection & $6(7)$ & $3(3)$ \\
Other & $36 \cdot 4(4-74)$ & $39 \cdot 3(3-81)$ \\
Mean age (range) (years) & & \\
\hline
\end{tabular}

TABLE II-Agreement between patients and experts on which events were related to treatment

\begin{tabular}{|c|c|c|c|c|c|}
\hline & \multirow{2}{*}{$\begin{array}{c}\text { No } \\
\text { of } \\
\text { events }\end{array}$} & \multicolumn{2}{|c|}{ No of events considered drug related } & \multirow{2}{*}{$\begin{array}{l}\text { Observed } \\
\text { agreement } \\
(\%)\end{array}$} & \multirow[b]{2}{*}{$x+$} \\
\hline & & Patients & Experts ${ }^{\star}$ & & \\
\hline Ears and hearing & 60 & & & 100 & \\
\hline Eyes and eyesight & 42 & & 5 & 88 & \\
\hline Nose and sense of smell & 68 & & & 100 & \\
\hline Mouth and sense of taste & 76 & 4 & 4 & 92 & $0 \cdot 21$ \\
\hline Throat & 77 & & & 100 & \\
\hline Chest and breathing & 74 & 1 & 1 & 100 & \\
\hline Heart & 8 & 1 & & 88 & \\
\hline Liver & 2 & & & 100 & \\
\hline Stomach & 49 & 10 & 9 & 78 & $0 \cdot 28$ \\
\hline Kidneys, bladder, and urine & 68 & & 2 & 97 & \\
\hline Head and face & 74 & 4 & 2 & 95 & $0 \cdot 31$ \\
\hline Neck and back & 46 & & 1 & 98 & \\
\hline Arms, hands, legs, and feet & 44 & 2 & & 95 & \\
\hline Skin & 26 & 2 & 14 & 54 & $0 \cdot 13$ \\
\hline Balance, mood, memory, and concentration & 71 & 3 & & 96 & \\
\hline Sleeping & 88 & 4 & 1 & 97 & $0 \cdot 39$ \\
\hline Appetite, thirst, and weight & 98 & & & 100 & \\
\hline Bowel function & 39 & 5 & 15 & 64 & $0 \cdot 13$ \\
\hline Sexual function & 9 & & & 100 & \\
\hline Total & 1019 & 36 & 54 & 94 & $0 \cdot 26$ \\
\hline
\end{tabular}

* Similar frequencies do not necessarily indicate concordance.

tWhen not enough events were considered to be drug related $x$ was not calculated.
TABLE III-Examples of calculation of $x$ statistic

\begin{tabular}{lccc}
\hline & \multicolumn{3}{c}{$\begin{array}{c}\text { Experts' opinion that event is related to } \\
\text { treatment }\end{array}$} \\
\cline { 2 - 4 } $\begin{array}{c}\text { Patient's opinion that event is } \\
\text { related to treatment }\end{array}$ & Yes & No & Total \\
\hline \multirow{4}{*}{$\begin{array}{l}\text { Ses } \\
\text { No }\end{array}$} & $\begin{array}{c}\text { Skin disorders } \\
\text { t }\end{array}$ & & \\
\hline Total & 12 & 12 & 24 \\
\hline & 14 & 12 & 26 \\
Yes & Sleep disorders $†$ & & \\
No & 1 & 3 & 4 \\
\hline Total & 1 & 84 & 84 \\
\hline
\end{tabular}

$\star$ Observed agreement $=0.54 ;$ expected agreement $=0 \cdot 47 ; x=0 \cdot 13$. tObserved agreement $=0.966 ;$ expected agreement $=0.944 ; x=0.39$.

TABLE IV-Performance of two types of report form in detecting "true" adverse drug reactions. Figures are expressed as percentages (95\% confidence intervals)

\begin{tabular}{lcc}
\hline & Event report & Reaction report \\
\hline Sensitivity & $42(25$ to 59$)$ & $24(13$ to 36$)$ \\
Specificity & $58(53$ to 62$)$ & $98(97$ to 99$)$ \\
Positive predictive value & $6(3$ to 9$)$ & $36(20$ to 52$)$ \\
Negative predictive value & $94(91$ to 97$)$ & $96(95$ to 97$)$ \\
\hline
\end{tabular}

of the patients who were interviewed. In general, patients who received the combined form and those who received only the reaction report form seemed to be similar.

Reliability and validity of event report form-The responses contained in 83 completed event report forms were analysed. There were $2654(97 \%)$ responses to 2739 statements; 419 (16\%) statements elicited responses indicating an abnormality or change in a body system. The agreement between what was reported in the event report forms and the response to the same statements posed to the same patients during the telephone interviews was $85 \%$. This suggested that the event report forms were reliable $(x=0.56)$. More positive responses were elicited by the supplementary questions during the telephone interviews, and this was reflected in the modest sensitivity of the event report form (54\%). Specificity was high (94\%) and if an event was noted on the form it was likely to be confirmed at telephone interview (positive predictive value $79 \%$ ). There was no appreciable difference when the data were analysed by sex and age.

Agreement between patients and expert panel-The agreement between the patients and the panel was assessed from the 1019 clinical events reported during the telephone interviews. Most of the events were related to the patients' illness. Overall the experts thought that more events were related to the treatment than did patients (table II). This was particularly so in the case of disorders of the skin (itching and rashes) and bowel (diarrhoea). In contrast, patients seemed as likely as the experts to attribute upper gastrointestinal disturbances to the drugs. The overall agreement between patients and panel was high owing to their agreement that most of the events were not related to treatment. As indicated by the $x$ statistic, agreement on whether disorders of the stomach and head and of sleep were related to treatment was fair. Table III gives examples of the calculation of the $x$ statistic.

Comparison of reaction reporting and event reportingThe event report form had a higher sensitivity than the reaction report form for the adverse reactions when compared with the experts' judgment on the complete sets of events reported (table IV). By contrast it had a lower specificity and a lower positive predictive value than the reaction report form. 


\section{Discussion}

Our primary interest in this study was whether patients could be persuaded to report their experiences during drug treatment in a reliable and valid way to a group other than those who prescribed the drug for them. The results suggest that patients will comply with such a request, though the response rate of $77 \%$ was achieved only after telephone reminders, and this might be impractical in a large study. The study conditions were optimal as patients were mainly young and receiving short term treatment with drugs with known adverse reactions.

Most of the events reported were related to the illness of the patient. This indicates the "noise" that should be expected in a system based on comprehensive reporting of events by patients. The low rate of "true" adverse drug reactions meant that the expected chance agreement between patients and experts that events were not related to treatment was high. This should not obscure the fact that patients were conservative in attributing clinical events to their treatment. Furthermore, as they did not agree with the experts that skin and bowel complaints were related to their treatment this suggests that their responses were not cued by advice given previously by family doctors or pharmacists. This conservatism in reporting reactions is important. A notable limitation of reaction reporting is its low sensitivity for detecting true adverse reactions. The higher sensitivity of event reporting probably shows that this is a better detection system, although the positive predictive value was low, partly because so many of the events were related to the patients' illness. The event rate was high among our patients, who were mostly suffering from acute infections, and it might be lower during monitoring of drugs used to treat other conditions.

Though direct participation of patients in event reporting has been described previously,${ }^{89}$ this is one of only two attempts to evaluate patients' reports of adverse reactions. ${ }^{67}$ As our conclusions differ from those of the study of Fisher et al the reasons require examination. Fisher et al described a study in the United States in which trained staff telephoned patients who had been identified as receiving a study drug. ${ }^{6}$ They compared the results of a simple open ended question about adverse experiences (analogous to our reaction reports) with systematic interviews conducted with each patient after the open ended question (analogous to our event reports). Using a measure of validity that they had designed, which we interpret as providing information similar to that provided by the positive predictive value, they showed that open ended questions were more likely than systematic questions to identify true reactions. On the basis of this they compared open ended questions initiated by the centre with reports of adverse clinical events initiated by the patients and made by free telephone calls to the monitoring centre. ${ }^{7}$ Concluding that the methods were equally likely to identify real reactions and that reports initiated by patients were easier to deal with, they recommended postmarketing surveillance based on reports initiated by patients. Our data also show that patients' reports of reactions have a higher positive predictive value than their reports of events, but we are concerned at the apparently low sensitivity of this system, which depends entirely on the initiative of the patient. Furthermore, the lack of agreement between patients and an expert panel in attributing adverse events to drug treatment is worrying and may indicate that voluntary reports from patients about known adverse reactions will be poor in quality. Possibly patients in the United States are better informed than those in Australia about which drug reactions to expect during treatment, which would improve the quality of their reporting. This might increase the level of agreement between the views of patients and experts. This would not, however, necessarily increase the ability of such a system to generate data about previously unsuspected adverse reactions to new drugs.

In the next few years patients will probably participate more in decisions regarding prescriptions and their knowledge of drugs will probably increase, particularly if they are provided with information leaflets. This welcome trend may be accompanied by requests from consumer groups for national monitoring centres to encourage patients to report directly suspected adverse reactions to drugs. We believe that national centres should resist this pressure as reporting of reactions by patients seems unlikely to contribute much to our knowledge of the effects of new drugs. Voluntary reports by health professionals to monitoring centres seldom identify previously unsuspected adverse reactions, but clinical details and comprehensive follow up information including laboratory results are essential for thorough evaluation of reactions and their cause. Our experience suggests that patients' reports of reactions will not contribute much to this.

Our data have shown the reliability and validity of written reporting of events. Although we are still not sure of the value of postmarketing surveillance based on patients' reports, we believe that a large scale trial of event reporting initiated by patients is justified. If event report forms were distributed routinely by community pharmacists thousands of reports could be obtained for widely used drugs. The reports would be much more efficiently handled if patients completed forms that could be processed by an automated reading system. If responses were available from patients receiving drugs with similar indications and possibly also from a population not taking any drugs the reports could be screened for clusters of events in particular systems of the body. The lack of detail would prevent much clinical interpretation, but the system would be cheap and might help the early identification of adverse reactions. If a new side effect was observed it could be confirmed or explained by using the conventional national monitoring system for voluntary reports from health professionals.

We acknowledge the help of the 20 community pharmacists and Dr John McEwen, medical officer and secretary of the Australian Adverse Drug Reactions Advisory Committee. This study was supported by a grant from the National Health and Medical Research Council of Australia.

1 Colin-Jones DG, Langman MJS, Lawson DH, Vessey MP. Postmarketing surveillance of the safety of cimetidine: mortality during second, third, and fourth years of follow up. Br Med $\mathcal{F} 1985 ; 291: 1084-8$.

2 Inman WHW. Postmarketing surveillance of adverse drug reactions in general practice. II. Prescription-event monitoring at the University of Southampton. Br.Med f 1981:282:1216-7.

3 Crombie IK, Brown SV, Hamley JG. Postmarketing drug surveillance by record linkage in Tayside. $\mathcal{F}$ Epidemiol Community Health 1984;38:226-31.

4 Strom BL, Carson JL, Morse ML, LeRoy AA. The computerized on-line Medicaid pharmacuetical analysis and surveillance system: a new resource for postmarketing drug surveillance. Clin Pharmacol Ther 1985;38:359-64.

Jick H, Madsen S, Nudelman PM, Perera DR, Stergachis A. Postmarketing Jick H, Madsen S, Nudelman PM, Perera DR, Stergachis A. Postmarketing
follow-up at group health cooperative of Puget Sound. Pharmacotherapy 1984;4:99-100.

6 Fisher S, Bryant SG, Kluge RM. Detecting adverse drug reactions in postmarketing surveillance: interview validity. Drug Information fournal 1987;21:173-83

7 Fisher S, Bryant SG, Solovitz BL, Kluge RM. Patient-initiated postmarketing surveillance: a validation study. $\mathcal{F}$ Clin Pharmacol 1987;27:843-54

8 Borden EK, Lee JG. A methodological study of post-marketing drug evaluation using a pharmacy-based approach. $\mathcal{f}$ Chronic Dis 1982;35:803-16.

Luscombe FA. Methodological issues in pharmacy-based post-marketing surveillance. Drug Information foumal 1985; 19:269-74.

10 Fleiss JL. Statistical methods for rates and proportions. 2nd ed. New York: Wiley, 1981:217-8.

Wiley, 1981:217-8.
11 Feinstein AR. Clinical biostatistics XXXI. On the sensitivity, specificity, and discrimination of diagnostic tests. Clin Pharmacol Ther 1975;17:104-16.

(Accepted 27 fune 1988) 